\title{
Análisis numérico de las especies de Prosopis L. (Fabaceae) de las costas de Perú y Ecuador
}

\section{Numerical analysis of Prosopis L. (Fabaceae) species from the coasts of Peru and Ecuador}

\author{
Alicia D. Burghardtt, ${ }^{1,2}$ M. Magdalena Brizuela ${ }^{1,2}$, M. Pía Mom ${ }^{1}$, Luis Albán ${ }^{3} y$ \\ Ramón A. Palacios ${ }^{1,2}$
}

\begin{abstract}
1 Departamento de Biodiversidad y Biología Experimental, Facultad de Ciencias Exactas y Naturales, Universidad de Buenos Aires. Pabellón $2-4^{\circ}$ piso - Laboratorio 11 nt. Güraldes y Costanera Norte. Int. Güiraldes y Costanera Norte. Ciudad Universitaria 1428, Buenos Aires. República Argentina. 2 Consejo Nacional de Investigaciones Científicas y Técnicas 3 Naturaleza y Cultura Internacional - Perú.

Email Alicia Burghardt: alibu@ bg.fcen.uba.ar

Email María Magdalena Brizuela: brizuela@bg.fcen.uba.ar

Email Ramón Palacios: palacios@ bg.fcen.uba.ar
\end{abstract}

Presentado: 22/03/2010 Aceptado: $\quad 24 / 09 / 2010$ Publicado online: 21/01/2011

\begin{abstract}
Resumen
Diferentes revisiones coinciden en señalar 2 o 3 especies de Prosopis para el sur de Ecuador y norte de Perú: P. juliflora (SW) DC, P. pallida (Humb. et Bonpl. ex Willd.) Kunth y P. affinis Sprengel. En el presente trabajo se informa del análisis cuantitativo de caracteres foliares de especímenes del genero Prosopis, recolectados a lo largo de la costa desde Arequipa (Perú) a Manta (Ecuador). Los resultados señalan tres grupos bien definidos. Del análisis comparativo de los tipos y ejemplares de herbario de todas las especies y sinónimos citados para la zona de estudio surge que los taxones existentes son: P. pallida, P. limensis Bentham, ambos de amplia distribución, y P. chilensis (Molina) Stuntz emend Burkart restringido al valle del río Camaná. Estos tres taxones se corresponden con los tres grupos obtenidos del análisis numérico. Debe señalarse la exclusión del área de $P$. juliflora y $P$. affinis. Se sugiere no utilizar las numerosas variedades señaladas para $P$. pallida.
\end{abstract}

Palabras claves: Caracteres foliares, análisis numérico, Prosopis, algarrobos, Algarobia, Fabaceae.

\section{Abstract}

To the south of Ecuador and northern Peru is indicated the presence of 2 or 3 species of Prosopis: P. juliflora (SW) DC, P. pallida (Humb. et Bonpl. ex Willd.) Kunth and $P$. affinis Sprengel. In this paper we report the results of quantitative analysis of leaf characters of specimens of the genus Prosopis, collected along the coast from Arequipa (Peru) to Manta (Ecuador). The results point out three well defined groups. When we compare all the species and synonymous mentioned for the study zone with our results, conclude that are: P. pallida, $P$. limensis Bentham (both with a wide distribution) and P. chilensis (Molina) Stuntz emend Burkart restricted to the Camana river valley. It is pointed out the exclusion from the area of $P$. juliflora and $P$. affinis. We suggested that the several varieties mentioned for $P$. pallida should not be used for the moment.

Keywords: Foliar characters, numerical analysis, Prosopis, algarrobos, Algarobia, Fabaceae.

\section{Introducción}

Los algarrobos, Prosopis L. (Fabaceae), de la costa peruanoecuatoriana han tenido varias revisiones, siendo las más recientes las de Burkart (1976), Ferreyra (1987), Díaz Celis (1995) y Pasiecznick et al. (2001); estos autores concuerdan que P. juliflora (SW) DC, y P. pallida (Humb. et Bonpl. ex Willd.) Kunth son las especies comunes en la zona. Ferreyra (1987), incluyó además a $P$. affinis Sprengel, aunque la presencia de esta última especie en la zona de estudio planteaba ciertas dudas, ya que su distribución, en la Cuenca del Plata (Uruguay, Argentina, Paraguay y Brasil), está asociada a un clima mucho más húmedo, por lo que Díaz Celis (1995) y Pasiecznick et al. (2001) la consideraron como una identificación incorrecta.

Mom et al. (2002) señalaron la presencia de P. pallida y $P$. $l i$ mensis Bentham en la Región Grau. Prosopis limensis fue incluida como sinonimia de P. pallida por Burkart (1976), sin embargo Mom et al. (2002) indican que existen las dos entidades y que se corresponden con los tipos de las especies antes citadas.

El presente trabajo es continuación del realizado por Mom et al. (2002); y realiza un análisis de las especies de algarrobos desde Tacna a Zarumilla en Perú, y en Ecuador desde Huasquillas a Manta; con la finalidad de estudiar la posible presencia de $P$. affinis y de $P$. juliflora en áreas en las cuales se señalaba su presencia. Se utilizan caracteres cuantitativos foliares, los que son considerados importantes y eficaces para distinguir las especies de Prosopis (Díaz Celis 1995, Harris et al. 2003); además los caracteres foliares pueden proporcionar una valiosa guía de campo para identificación de especies (Pasiecznick et al. 2001)

\section{Material y métodos}

Material estudiado.- Se realizó un viaje de colección desde Tacna (Perú) a Manta (Ecuador) en febrero de 2001, con el fin de obtener muestras de Prosopis (ejemplares de herbario). En la Tabla 1 se indican la información de las muestras utilizadas y los herbarios donde están depositadas: Herbario del Departamento de Ciencias Biológicas de la Facultad de Ciencias Exactas y Naturales de la Universidad de Buenos Aires (BAFC) y Herbarium Truxillense de la Universidad Nacional de Trujillo (HUT).

Además se examinó material de varios herbarios, que no fueron incluidos en el análisis numérico:

\section{Prosopis pallida}

Perú:

Tipo: América Meridional. Perú Humboldt \& Bonpland s/n (Foto Herbario Willdenow 19131, Museo Botanicum Berolinense B).

Dpto. Amazonas. Prov. Bagua, P. Hutchinson 1501 (F); Caserío Shuape. Ferr. Holle, Viceland, Chicoana 20581. 16/X, 1986 (USM).

Dpto. Piura, Prov. Paita. 3 a 4 km E of Talara. 20 msn. O. Horton 11587. 4/IV/1939 (F); Prov. Piura, Talara. J. Campos s/n, 1/X/1981 (USM); Santa Clara, Sechura, Plaza de Armas. R. Ferreyra - J. Vilela 20041, 20042 (USM); Prov. Sullana, Sullana, campo reforestación "El Algarrobo". R. Ferreyra, Aguilar 19319 (USM); "Savana". R. Ferreyra 19143 A (USM); "El Algarrobo", S. Valdivia - A. Montesinos 6. 19///1979 (USM); "El Algarrobo", R. Ferreyra 19125 (USM).

Dpto. Lima, Parque Montero, F. Encarnación 163 (USM).

Dpto. Lambayeque, Prov. Lambayeque. Distrito Olmos, Racalí. V. Chavez s/n (USM). A.Weberbauer 6172 (F). Negritos. Oscar Haught 1928 (F).

Dpto. Tumbes, cerca de Bocapán. R. Ferreyra, E. Cerrate, O. Tovar 10555. 24/IV/1955 (MO); Prov. Tumbes R. Lao s/n (F). 
Tabla 1. Información de las muestras utilizadas en el análisis de taxonomía numérica de las especies de Prosopis L. (Fabaceae) de las costas de Perú y Ecuador, se indican los herbarios donde están depositadas.

\begin{tabular}{|c|c|c|c|c|c|}
\hline Pais & Localidad & Lugar & Coordenadas & Fecha & Número de muestra (Herbario) \\
\hline \multirow{15}{*}{ 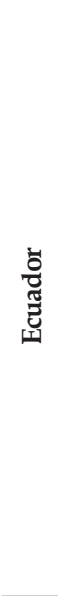 } & Guayas & Colonche & & $16 / 02 / 2002$ & 3306 (BAFC,HUT) \\
\hline & Manabí & Río Chico a Rocafuerte & & $15 / 02 / 2002$ & 3302 (BAFC,HUT) \\
\hline & Manabí & Rocafuerte a Manta & & $15 / 02 / 2002$ & 3303, 3304 (BAFC,HUT) \\
\hline & Manabí & Machalilla & & $16 / 02 / 2002$ & 3305 (BAFC,HUT) \\
\hline & Loja & Casanga empalme & & $19 / 02 / 2002$ & 3311 (BAFC,HUT) \\
\hline & Loja & Sabanilla a Zapatillo & & $20 / 02 / 2002$ & 3312 (BAFC, HUT) \\
\hline & Loja & Sabanilla a La Ceiba & & $20 / 02 / 2002$ & 3313, 3314 (BAFC, HUT) \\
\hline & Loja & La Ceiba & & $20 / 02 / 2002$ & 3315, 3316 (BAFC, HUT) \\
\hline & DelOro & Huaquilla, Santa Rosa. & & $13 / 02 / 2002$ & 3293, 3294 (BAFC, HUT) \\
\hline & Guayas & Puerto Inca, Guayaquil. & & $13 / 02 / 2002$ & 3295, 3296 (BAFC, HUT) \\
\hline & Guayas & Guayaquil-Santa Elena. & & $14 / 02 / 2002$ & 3297, 3298 (BAFC, HUT) \\
\hline & Manabi & Pto. Cayo-Jipijapa. & & $15 / 02 / 2002$ & 3299 (BAFC, HUT) \\
\hline & Manabi & Río Chico-Rocafuerte. & & $15 / 02 / 2002$ & 3300, 3301 (BAFC, HUT) \\
\hline & Loja & Catamayo, La Toma. & & $19 / 02 / 2002$ & 3307 (BAFC, HUT) \\
\hline & Loja & Casanga, cruzando el río Playas. & & $19 / 02 / 2002$ & 3308, 3309, 3310 (BAFC, HUT) \\
\hline \multirow{38}{*}{ 遶 } & Tumbes & Alrededores de Puerto Pizarro. & $3^{\circ} 30^{\prime} 49^{\prime \prime} \mathrm{S} 80^{\circ} 23^{\prime} 06^{\prime \prime} \mathrm{W}$ & $21 / 02 / 2010$ & $3110(\mathrm{BAFC})$ \\
\hline & Piura & $\begin{array}{l}\text { A } 15 \mathrm{~km} \text { del caserío Santa Ana, carretera a Piura, margen } \\
\text { izquierda del río Piura. }\end{array}$ & $4^{\circ} 56^{\prime} 42^{\prime \prime} \mathrm{S} 80^{\circ} 22^{\prime} 10^{\prime \prime} \mathrm{W}$ & $21 / 02 / 2010$ & 3101 (BAFC) \\
\hline & Piura & Tambo Grande, camino al caserío Locuto, Vega de la Ardilla & $4^{\circ} 57^{\prime} 37^{\prime \prime} \mathrm{S} \quad 80^{\circ} 23^{\prime} 47^{\prime \prime} \mathrm{W}$ & $21 / 02 / 2010$ & 3098 (BAFC) \\
\hline & Piura & $\begin{array}{l}\text { Tambo Grande, camino al caserío El Carmen, comunidad } \\
\text { campesina San Juan Bautista de Locuto, km } 21 \text { de antigua } \\
\text { carretera Panamericana. }\end{array}$ & $4^{\circ} 59^{\prime} 37^{\prime \prime} \mathrm{S} 80^{\circ} 23^{\prime} 47^{\prime \prime} \mathrm{W}$ & $23 / 02 / 2010$ & 3097 (BAFC) \\
\hline & Piura & Colán, Iglesia de Colán. & $5^{\circ} 00^{\prime} 13^{\prime \prime} \mathrm{S} 81^{\circ} 03^{\prime} 18^{\prime \prime} \mathrm{W}$ & $18 / 02 / 2010$ & 3065 (BAFC) \\
\hline & Piura & Paita, Centro Recreativo del Club de Leones. & $5^{\circ} 05^{\prime} 40^{\prime \prime} \mathrm{S} 81^{\circ} 04^{\prime} 39^{\prime \prime} \mathrm{W}$ & $19 / 02 / 2010$ & 3064 (BAFC) \\
\hline & Piura & Caserío Santa Rosa del 32. & $5^{\circ} 08^{\prime} 13^{\prime \prime} \mathrm{S} \quad 80^{\circ} 21^{\prime} 05^{\prime \prime} \mathrm{W}$ & $21 / 02 / 2010$ & 3095 (BAFC) \\
\hline & Piura & Castilla, antigua panamericana Chiclayo - Piura Km 235. & $5^{\circ} 09^{\prime} 20^{\prime \prime} \mathrm{S} 80^{\circ} 22^{\prime} 09^{\prime \prime} \mathrm{W}$ & $21 / 02 / 2010$ & 3096 (BAFC) \\
\hline & Piura & Antigua Panamericana Chiclayo - Piura. Km 202. & $5^{\circ} 10^{\prime} 00^{\prime \prime} \mathrm{S} 80^{\circ} 08^{\prime} 55^{\prime \prime} \mathrm{W}$ & $21 / 02 / 2010$ & 3094 (BAFC) \\
\hline & Piura & Cruce El Trébol, carretera Piura-Paita-Sullana. & $5^{\circ} 10^{\prime} 03^{\prime \prime} \mathrm{S} \quad 80^{\circ} 41^{\prime} 18^{\prime \prime} \mathrm{W}$ & $18 / 02 / 2010$ & 3073 (BAFC) \\
\hline & Piura & Cruce El Trébol, carretera Piura-Paita-Sullana. & $5^{\circ} 10^{\prime} 03^{\prime \prime} \mathrm{S} \quad 80^{\circ} 41^{\prime} 18^{\prime \prime} \mathrm{W}$ & $18 / 02 / 2010$ & 3072 (BAFC) \\
\hline & Piura & Piura, campus de la Universidad de Piura. & $5^{\circ} 10^{\prime} 16^{\prime \prime} \mathrm{S} 80^{\circ} 38^{\prime} 06^{\prime \prime} \mathrm{W}$ & $17 / 02 / 2010$ & 3059,3062 (BAFC) \\
\hline & Piura & Piura, campus de la Universidad de Piura. & $5^{\circ} 10^{\prime} 16^{\prime \prime} \mathrm{S} 80^{\circ} 38^{\prime} 06^{\prime \prime} \mathrm{W}$ & $17 / 02 / 2010$ & $3058,3060,3061$ (BAFC) \\
\hline & Piura & Chulucanas, camino a Caserío Alto el Gallo & $5^{\circ} 11^{\prime} 05^{\prime \prime} \mathrm{S} 80^{\circ} 11^{\prime} 08^{\prime \prime} \mathrm{W}$ & $21 / 02 / 2010$ & $3092(\mathrm{BAFC})$ \\
\hline & Piura & $\begin{array}{l}\text { Morropón, La Matanza, antigua Panamericana carretera } \\
\text { Chiclayo - Piura km } 188 .\end{array}$ & $5^{\circ} 16^{\prime} 16^{\prime \prime} \mathrm{S} 80^{\circ} 05^{\prime} 53^{\prime \prime} \mathrm{W}$ & $20 / 02 / 2010$ & 3089 (BAFC) \\
\hline & Piura & Sechura, carretera a manglar de San Pedro. & $5^{\circ} 20^{\prime} 09^{\prime \prime} \mathrm{S} 80^{\circ} 51^{\prime} 12^{\prime \prime} \mathrm{W}$ & $19 / 02 / 2010$ & 3075 (BAFC) \\
\hline & Piura & Dist. Vice, Manglar de San Pedro. & $5^{\circ} 30^{\prime} 58^{\prime \prime} \mathrm{S} 80^{\circ} 53^{\prime} 21^{\prime \prime} \mathrm{W}$ & $19 / 02 / 2010$ & 3074 (BAFC) \\
\hline & Piura & Dist. Sechura, Plaza de Armas, cerca del local parroquial. & $5^{\circ} 33^{\prime} 15^{\prime \prime} \mathrm{S} \quad 80^{\circ} 49^{\prime} 15^{\prime \prime} \mathrm{W}$ & $19 / 02 / 2010$ & 3076 (BAFC) \\
\hline & Piura & Inmediaciones de la ciudad de Sechura. & $5^{\circ} 33^{\prime} 18^{\prime \prime} \mathrm{S} 80^{\circ} 49^{\prime} 02^{\prime \prime} \mathrm{W}$ & $19 / 02 / 2010$ & 3077, 3078 (BAFC) \\
\hline & Piura & Caserío Belisario. & $5^{\circ} 50^{\prime} 36^{\prime \prime} \mathrm{S} 80^{\circ} 26^{\prime} 50^{\prime \prime} \mathrm{W}$ & $20 / 02 / 2010$ & 3083, 3084 (BAFC) \\
\hline & Piura & Carretera Chiclayo - Bayovar. & $5^{\circ} 50^{\prime} 55^{\prime \prime} \mathrm{S} 80^{\circ} 58^{\prime} 55^{\prime \prime} \mathrm{W}$ & $19 / 02 / 2010$ & 3079 (BAFC) \\
\hline & Piura & Carretera Sechura - Bayovar Km 33. & $5^{\circ} 51^{\prime} 05^{\prime \prime} \mathrm{S} 80^{\circ} 53^{\prime} 31^{\prime \prime} \mathrm{W}$ & $19 / 02 / 2010$ & 3080, 3081, 3082 (BAFC) \\
\hline & Piura & $\begin{array}{l}\text { Olmos, caserío Ancol Chico, margen derecha del río Cascajal, } \\
\text { camino a Olmos. }\end{array}$ & $5^{\circ} 54^{\prime} 35^{\prime \prime} \mathrm{S} \quad 80^{\circ} 00^{\prime} 23^{\prime \prime} \mathrm{W}$ & $20 / 02 / 2010$ & 3086, 3087 (BAFC) \\
\hline & Piura & $\begin{array}{l}\text { Caserío Cerro de Arena, margen derecha del río Cascajal, } \\
\text { camino a Olmos. }\end{array}$ & $5^{\circ} 55^{\prime} 17^{\prime \prime} \mathrm{S} 80^{\circ} 12^{\prime} 10^{\prime \prime} \mathrm{W}$ & $20 / 02 / 2010$ & 3085 (BAFC) \\
\hline & Piura & Piura. Sullana km 1007. & & $12 / 02 / 2002$ & 3287 (BAFC, HUT) \\
\hline & Piura & Ruta a Sullana Paita km 16. & & $18 / 02 / 2001$ & 3068 (BAFC, HUT) \\
\hline & Piura & Las Lomas km. 1026. & & $12 / 02 / 2002$ & 3289, 3290, 3292 (BAFC, HUT) \\
\hline & Piura & La Tina. & & $21 / 02 / 2002$ & 3317 (BAFC, HUT) \\
\hline & Piura & Piura Sullana km 1007. & & $12 / 02 / 2002$ & 3288. (BAFC, HUT) \\
\hline & Piura & Las Lomas km 1026. & & $12 / 02 / 2002$ & 3291. (BAFC, HUT) \\
\hline & Ancash & Entrada a Huarmey. & & 08/02/2002 & 3284. 3285 (BAFC, HUT) \\
\hline & Ancash & Entrada a Huarmey II/2002. & & $08 / 02 / 2002$ & 3283 (BAFC, HUT) \\
\hline & Ancash & Casma, puente Carrizal. & & 08/02/2002 & 3286 (BAFC, HUT) \\
\hline & Ica & Valle río Poroma, ruta $5 \mathrm{~km} \mathrm{467,} \mathrm{sur} \mathrm{de} \mathrm{Nazca.}$ & & $07 / 02 / 2002$ & 3280 (BAFC, HUT) \\
\hline & Ica & Valle río Poroma, ruta $5 \mathrm{~km} \mathrm{466,} \mathrm{sur} \mathrm{de} \mathrm{Nazca.}$ & & $08 / 02 / 2002$ & 3281 (BAFC, HUT) \\
\hline & Ica & 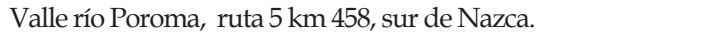 & & $08 / 02 / 2002$ & 3282. (BAFC, HUT) \\
\hline & Arequipa & Prov. Castilla, Punta Colorada, Río Majes. & & 07/02/2002 & 3274, 3275, 3276 (BAFC, HUT) \\
\hline & Arequipa & 10 km al norte de Camaná, Paso Hawai. & & $07 / 02 / 2002$ & 3277,3278(BAFC,HUT) \\
\hline
\end{tabular}




\section{Ecuador:}

Prov. Loja, on the Catacocha-Macará, C. $7 \mathrm{~km} \mathrm{~N}$ of the cross-road to Celica, 600 msn. G. Harling, L. Anderson 18199, 12/IV/1980 (MO); Macará, J. Hart 1099 (MO); La Toma, Anne Macey 118, VII/1975 (LOJA); La Toma, 1400 msm, R. Espinosa 491, 5/VI/1946 (LOJA); $8 \mathrm{~km}$. de Zapotillo $4^{\circ} 18^{\prime} \mathrm{S}$ y $80^{\circ} 14 \mathrm{~W}$, A. Zamaniego y F. Vivar 30 (LOJA); Gonzanamá, Nambacola, El Húmedo 4²'47"S y 79³0'48”W, 4/II/1980, F. Vivar 1119 (LOJA); Macará- Zapotillo, Km. 13, 700-750 msm 4¹9'93"S y 7959'89'W, G. Lewis, P. Lozano 3048 III/1997, (LOJA); Celica, Quebrada Yaraco, 48'35"S y 7950'20"W, L. Emperaire 1224 (LOJA).

Prov. Guayas, Ancón, G. Harling, G. Storm \& B. Strön 8818, 29-30/ IV/1968 (MO); Santa Elena 18/11/1974, Al Gentry 10017 (MO, NY).

Prov. Manabí, Bahía de Caráquez, G. Harling, G. Storm \& B. Ström 9453 (MO).

Prov. Oro, Machala, Playa Los Cocos, Luz F. Vivar 716 (LOJA).

\section{Prosopis limensis}

\section{Perú:}

Tipo: Perú, Lima et Perú septentrionalis. Cuming 974 (K) (foto US ex K).

Río Apurimac, A. Weberbauer 5366 (F), 5907 (F).

Yautan, Mac Bride y Featherstone 2563 (F).

Dpto. Ica, Prov. Nazca, bosque de Jumana. J. Aguilar Gallardo s/n (USM); Prov. Ica, Pampas de Ocucaje, R. Ferreyra 1382 (USM).

Dpto. Piura Prov. Sullana, "EI Algarrobo", S. Valdivia -A. Montesinos 1,3 (USM); R. Ferreyra 19122 (USM).

Dpto. Arequipa, Prov. Islay, Loma de Mollendo, R. Ferreyra 12072 (USM).

Dpto. Tumbes, Prov. Tumbes, Punta Mal Pazo, R. Ferreyra 18966 (USM); Prov. Zarumillo, entre Zarumillo y El Salto, R. Ferreyra 10749, 29/IV/1955 (MO).

\section{Prosopis chilensis}

\section{Perú:}

Dpto Cuzco, Prov. Anta Sisal Cumyacc, C. Vargas 007410 (USM); Prov. La Convención, valley of Río Vilcanota near Quillabamba, 1010 msn. Y. Mescia 8026. 9/05/1938 (F).

Dpto. Arequipa, Prov. Castilla. Isla el rescate, antes de Aplao, Aguilar Gallardo s/n (USM); Aplao, "cultivado", Jaime Aguilar Gallardo s/n 11/XII/1982. (USM); Aplao, Lomas cerca de las chacras de arroz, José Aguilar Gallardo s/n 11/XII/1982 (USM).

Aplao B. Simpson 8575 A. (USM); Castilla, 10 Km. N Aplao, B. Simpson 86753 17/I/1977 (USM)

Hda. Potrero, C. Vargas 2041, 8/VIII/1940 (USM); A. Weberbauer $5919(\mathrm{~F})$.

\section{Métodos}

Los ejemplares colectados fueron analizados registrando los siguientes caracteres foliares: longitud total de la hoja $(\mathrm{cm})$; longitud del pecíolo $(\mathrm{cm})$; número de pares de pinas primarias; longitud de la pina primaria $(\mathrm{cm})$; ancho de la pina $(\mathrm{cm})$, número de pares de folíolos de la pina; distancia entre folíolos $(\mathrm{mm})$; largo del folíolo $(\mathrm{mm})$ y ancho del folíolo $(\mathrm{mm})$.

Se realizaron 10 mediciones en cada individuo y con estos datos se calculó la media por individuo para cada uno de los caracteres. Con ella, se construyó una matriz básica de datos de individuos por caracteres. Sobre la base de esta última se construyó una matriz de distancias (Cuadrado de las Distancias Euclideas) y se aplicó el método de agrupamiento de medias no ponderadas (UPGMA). Los análisis numéricos fueron realizados utilizando el paquete estadístico STATISTICA.

\section{Resultados}

Las medias por individuo se consignan en el Apéndice 1. La Figura 1 muestra el fenograma obtenido mediante el UPGMA.
Se observan claramente 3 grupos: A, B y C. El B1, se une al A-B y corresponde a materiales colectados en regiones del norte de Ecuador con mayores precipitaciones.

Los clusters A y B corresponden respectivamente a P. limensis y $P$. pallida. El cluster $\mathrm{C}$ agrupa a un número de individuos identificables como $P$. chilensis.

Esta información permite establecer una clave dicotómica para separar los taxones:

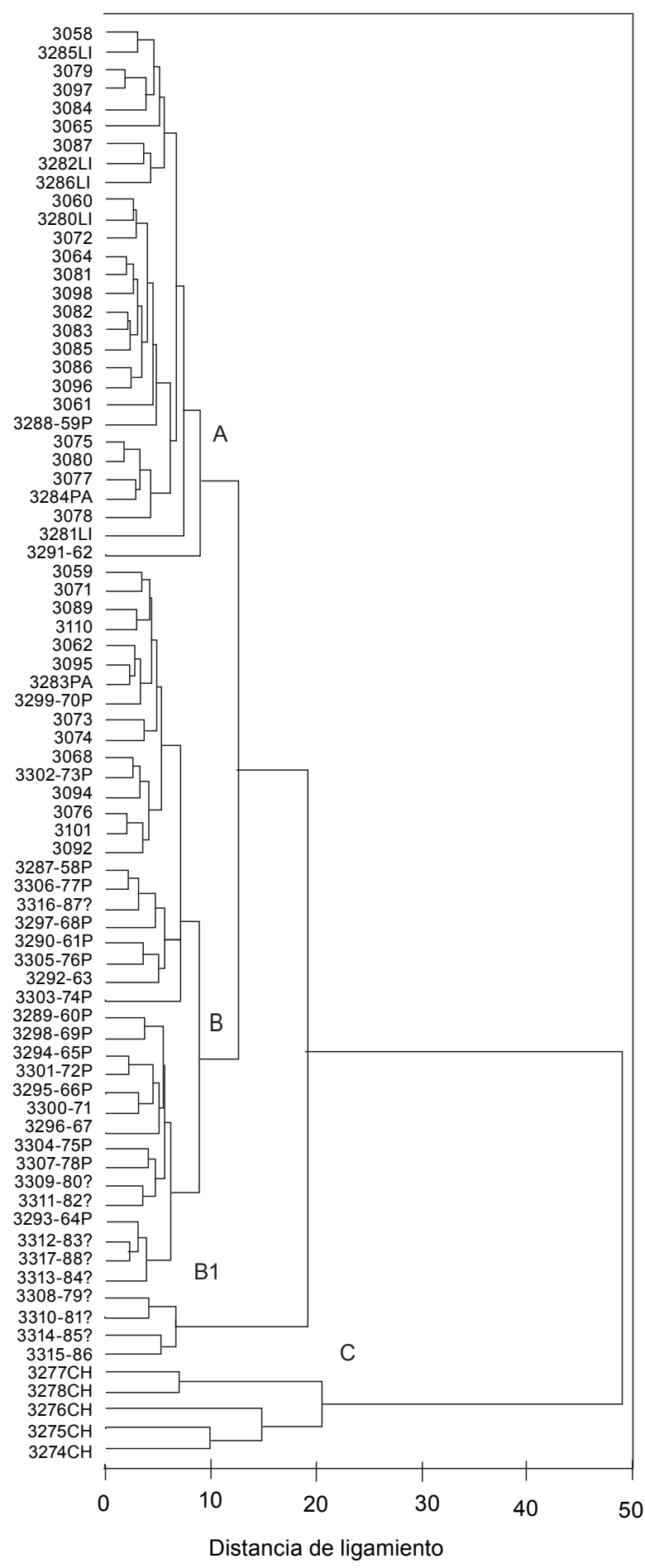

Figura 1. Fenograma construido con los caracteres foliares de Prosopis recolectados en la costa del norte de Perú y sur de Ecuador. Cuadrado de las Distancias Euclideanas, método de agrupamiento UPGMA. 
A.- Folíolos en general menores de $12 \mathrm{~mm}$ de longitud, pubérulos, distancia entre folíolos 1,0-4,4 $\mathrm{mm}$.

B.- Árbol con ramas principales algo fastigiadas ( $45^{\circ} \mathrm{o}$ menos), ramas terminales casi rectas, horizontales; braquiblastos poco desarrollados, con pocas hojas (1-3); hojas en general mayores de $6 \mathrm{~cm}$ long; folíolos en general mayores de $7 \mathrm{~mm}$ long $(7-10 \mathrm{~mm})$.

1. P. pallida

B”.- Árbol con ramas principales a $45^{\circ}$ o más, ramas terminales flexuosas, algo péndulas, braquiblastos muy desarrollados, con muchas hojas (3-10); hojas en general menores de $6 \mathrm{~cm}$ long.; folíolos en general menores de $7 \mathrm{~mm}$ long. 2. P. limensis

A".- Folíolos en general mayores de $12 \mathrm{~mm}$ de longitud, glabros, distancia entre folíolos 3,4-6,8 mm

3. P. chilensis

Prosopis pallida (Humb. et Bonpl. ex Willd.) Kunth. 1823. Nov. Gen. sp. Pl. 6: 306.

Iconografía: Mom et al. 2002.

Nombre común: "Algarrobo cholo". No existe información que introduzca cambios en la descripción publicada por Mom et al. (2002)

Prosopis limensis Bentham 1842. Hooker's Jour. Bot. 4: 350

Iconografía: Mom et al. 2002.

Nombre común: "Algarrobo zambo". Al igual que la especie anterior no existe información adicional sobre la descripción. Existe la posibilidad que puedan aparecer individuos híbridos entre $P$. pallida y $P$. limensis, tal como lo indica la posición del ejemplar 3291 de la Figura 1.

Prosopis chilensis (Molina) Stunz emend. Burkart 1940, Darwiniana 4: 105.

Nombre común: "Algarrobo". El aspecto general y de fruto pueden observarse en la Figura 2. Descripción: Árbol de hasta 10 metros de altura. Sin espinas en ramas viejas. Hojas 1-2 yu-

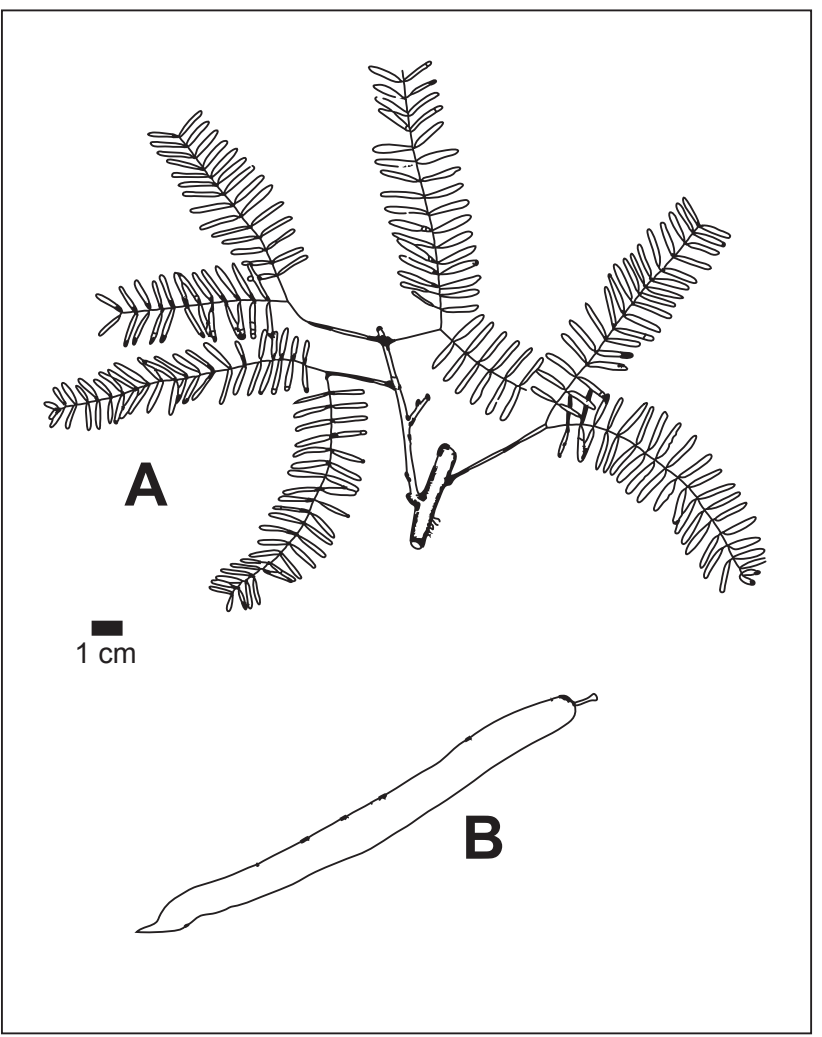

Figura 2. Prosopis chilensis A: aspecto general, $\mathrm{x}=1 \mathrm{~cm}$.; $\mathrm{B}$ fruto, $\mathrm{x}=1 \mathrm{~cm}$ gadas, pinnas 10,94-13,25 cm long. y 2,94-5,34 cm lat, pinnas generalmente uniyugadas, folíolos 2,87 a $5,45 \mathrm{~cm}$ long. y 1,54 a 2,2 $\mathrm{mm}$ lat. Inflorescencia en racimo espiciforme. Legumbre falcada a semianular con los márgenes paralelos o bien algo ondulados, color pajizo a veces con manchas violáceas de 16,2 a $25 \mathrm{~cm}$ x 1,24 a $1,75 \mathrm{~cm}$, mesocarpo dulce, palatable.

Distribución geográfica: En Perú, se encuentra además en el área de Cuzco; en Bolivia en la región oriental de la Precordillera Andina; en Argentina en el centro oeste y en Chile en el Valle del Elqui y en las inmediaciones de Santiago.

\section{Discusión}

Los ejemplares estudiados se reúnen en tres grupos definidos, los cuales pueden ser asignados a $P$. limensis (A), P. pallida (B) y $P$. chilensis (C) (Fig. 1). Los ejemplares 3308, 3310, 3314 y 3315 (de Ecuador, Prov. Loja), fueron colectados en la fracción del Bosque seco con predominio de Ceiba (en general por encima de los $500 \mathrm{~m}$ de altitud). En esta región las lluvias son algo superiores al bosque seco denominado Sapotal-Algarrobal y esto influiría en el mayor tamaño de los folíolos.

Díaz Celis (1995) presentó una clave para diferenciar los algarrobos del norte peruano. Según nuestro criterio lo atribuido por Díaz Celis (1995) a P. pallida debe ser designado como $P$. limensis y lo denominado $P$. juliflora, como $P$. pallida. Debe señalarse que este autor no indica los ejemplares estudiados. Por el momento y hasta disponer de pruebas experimentales, sobre la herencia de los caracteres que las determinan, sería mejor no utilizar las variedades propuestas por Díaz Celis (1995).

Prosopis juliflora no fue encontrada en el desierto costero y no hay ejemplares en los herbarios que avalen su presencia. Ferreyra (1987) señala que $P$. juliflora no existiría en Perú y que su binomio no corresponde a alguna de las especies del norte peruano; sin embargo la incluye con la variedad horrida. Estos materiales deben referirse a $P$. pallida.

El ejemplar 3291, morfológicamente afín a $P$. pallida, se separa del grupo B (P. pallida). La observación detallada de los materiales, sugiere que podría haberse originado de la hibridación de $P$. pallida $\times$ P. limensis (el orden es arbitrario).

La distribución de $P$. limensis es amplia en la zona costera peruana y de acuerdo a lo observado durante los viajes de recolección, esta especie sería la más resistente a la sequía.

Las colecciones realizadas para el presente trabajo y las revisiones de los herbarios no avalan la presencia de $P$. affinis en el desierto costero. Los materiales que Ferreyra (1987) cita como P. affinis tienen como característica común el color del fruto violáceo hasta morado, éste es un atributo que presenta tanto P. pallida como P. limensis.

Según nuestro criterio, los materiales citados como $P$. affinis por Ferreyra (1987) deben ser considerados como se indica a continuación:

\section{Prosopis pallida:}

Tumbes: Contralmirante Villar, cerca Bocapán, Ferreyra, Cerrate \& Tovar 10555 (USM); Zarumilla, Puerto Pizarro, Cerrate 4962 (USM).

Piura: Paita entre El Alto y Talara, Ferreyra 10757 (USM). Sullana "El Algarrobo", cerca Sullana, Ferreyra \& Aguilar 19119 (USM), 19121 (USM), 19134 (USM), 19137 (USM), 19139 (USM), 19317 (USM), Valdivia \& Montesinos 04 (USM); Marcavelica a $2 \mathrm{~km}$. G. Domínguez 09 (USM). 


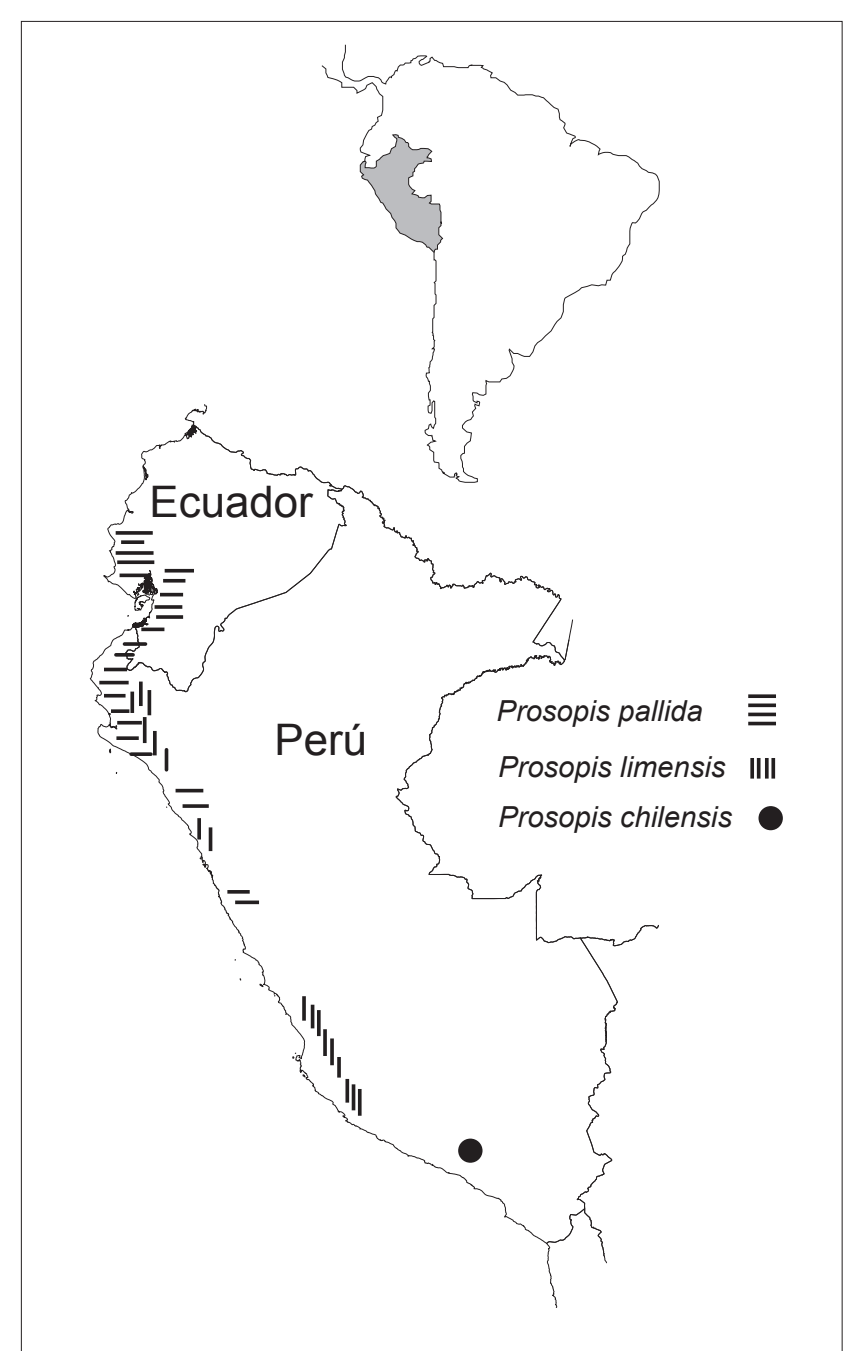

Figura 3. Mapa señalando la distribución de las especies de algarrobo en la zona costera peruano-ecuatoriana.

\section{Prosopis limensis:}

Tumbes: entre Zarumilla y El Salto, Ferreyra, Cerrate \& Tovar 10749 (USM); Tumbes Manglar Cherre, Ferreyra, Macedo \& Revilla 18966 (USM)

Ica: Ica, alrededores de la Laguna de La Huega, Ferreyra 8206 (USM), Laguna San Pedro, A. Luna s/n (USM), Laguna Huacachina, J. Revilla s/n (USM), Laguna Orovilca, A. Luna s/n (USM), Pampa de Ocucaje, Ferreyra 1382 (USM).
Se sugiere no seguir utilizando la denominación de las variedades de $P$. pallida (Ferreyra 1987) hasta la comprobación experimental de que los caracteres que las delimitan son el producto de variabilidad genotípica y no la respuesta a variaciones ambientales.

En el desierto costero, $P$. chilensis sólo fue coleccionada en las proximidades de Aplao y en la desembocadura del Río Camaná. Es posible que esta especie haya llegado a la región como una planta cultivada, en épocas previas a la colonización europea.

El presente trabajo concluye que:

a. No existen evidencias que avalen la presencia de Prosopis juliflora ni la de Prosopis affinis.

b. Los taxones existentes en la región costera peruanoecuatoriana son: Prosopis pallida (Humb. et Bonpl. ex Willd.) Kunth, Prosopis limensis Bentham, ambos de amplia distribución, y Prosopis chilensis (Molina) Stuntz emend Burkart restringido al valle del río Camaná. Estos tres taxones se corresponden con los tres grupos obtenidos del análisis numérico. La distribución de los algarrobos en la zona estudiada se muestra en la Figura 3.

\section{Literatura citada}

Burkart A. 1976. A monograph of the genus Prosopis (Leguminosae Subfam Mimosoideae). Journal of the Arnold Arboretum 57:219-249; 450-525

Díaz-Celis A. 1995. Los algarrobos. CONCYTEC. Lima, Perú. 217 págs.

Ferreyra R. 1987. Estudios sistemáticos de los algarrobos de la costa norte de Perú. Dirección de Investigación Forestal y Fauna. Ministerio de Agricultura, Lima, Perú. 31 págs.

Harris P.J.C., N.M. Pasiecznik, S.J. Smith, J.M. Billington, \& L. Ramírez. 2003. Differentiation of Prosopis juliflora (Sw.) DC. and P. pallida (H. \& B. ex. Willd.) H.B.K. using foliar characters and ploidy. Forest Ecology and Management 180(1): 153-164.

Mom M.P., A.D. Burghardt, R.A. Palacios \& L. Alban. 2002. Los algarrobos peruanos: Prosopis pallida y su delimitación. Arnaldoa 9(1): 39-48

Pasiecznick N.M., P. Felker, P.J.C. Harris, , Harsh, L. N., Cruz, G., Tewari, J. C., Cadoret, K. \& Maldonado, L.J. 2001. The Prosopis juliflora- Prosopis pallida Complex. A monograph. HDRA Coventry, UK pp. 162.

Apéndice 1. Matriz de datos. Promedios de los caracteres por individuo.

\begin{tabular}{|c|c|c|c|c|c|c|c|c|c|}
\hline $\mathrm{N}^{\circ}$ & $\begin{array}{l}\text { Longitud } \\
\text { total de la } \\
\text { hoja }(\mathrm{cm})\end{array}$ & $\begin{array}{l}\text { Longitud } \\
\text { del peciolo } \\
\text { (cm) }\end{array}$ & $\begin{array}{l}\mathrm{N}^{\circ} \text { pares } \\
\text { de pinnas } \\
\text { primarias }\end{array}$ & $\begin{array}{c}\text { Longitud } \\
\text { de la } 1^{\circ} \\
\text { pinna }(\mathrm{cm})\end{array}$ & $\begin{array}{l}\text { Ancho de } \\
\text { la } 1^{\circ} \text { pinna } \\
(\mathrm{cm})\end{array}$ & $\begin{array}{l}\mathrm{N}^{\circ} \text { pares } \\
\text { de foliolos } \\
1^{\circ} \text { pinna }\end{array}$ & $\begin{array}{l}\text { Dist entre } \\
\text { foliolos } \\
(\mathrm{mm}) 1^{\circ} \\
\text { pinna }\end{array}$ & $\begin{array}{l}\text { Largo de } \\
\text { foliolo } \\
(\mathrm{mm}) 1^{\circ} \\
\text { pinna }\end{array}$ & $\begin{array}{l}\text { Ancho de } \\
\text { foliolo } 1^{\circ} \text { pinna } \\
(\mathrm{mm})\end{array}$ \\
\hline 3058 & 3,65 & 0,90 & 2,00 & 1,90 & 0,70 & 8,75 & 1,65 & 4,50 & 1,60 \\
\hline 3059 & 8,00 & 1,78 & 2,50 & 3,81 & 1,45 & 10,13 & 3,39 & 7,31 & 2,44 \\
\hline 3060 & 5,10 & 1,00 & 3,00 & 3,00 & 0,95 & 10,75 & 2,00 & 5,60 & 1,66 \\
\hline 3061 & 4,90 & 1,70 & 2,50 & 1,40 & 0,80 & 10,50 & 3,12 & 5,60 & 1,75 \\
\hline 3062 & 5,60 & 1,30 & 2,00 & 4,40 & 1,50 & 10,00 & 3,25 & 7,25 & 2,00 \\
\hline 3064 & 5,10 & 1,18 & 2,00 & 2,78 & 1,14 & 10,38 & 3,13 & 5,58 & 1,36 \\
\hline 3065 & 5,00 & 0,60 & 1,75 & 2,06 & 0,83 & 10,25 & 2,00 & 4,25 & 2,13 \\
\hline 3068 & 6,98 & 1,76 & 2,25 & 3,94 & 1,49 & 11,75 & 3,25 & 7,50 & 2,20 \\
\hline 3071 & 7,30 & 2,28 & 2,00 & 4,45 & 1,29 & 10,00 & 4,18 & 7,31 & 2,44 \\
\hline \multirow[t]{2}{*}{3072} & 4,15 & 1,00 & 2,00 & 2,85 & 1,01 & 10,63 & 2,35 & 5,68 & 1,69 \\
\hline & & & & & & & & & (Continúa....) \\
\hline
\end{tabular}


Apéndice 1. Continuación.

\begin{tabular}{|c|c|c|c|c|c|c|c|c|c|}
\hline $\mathrm{N}^{\circ}$ & $\begin{array}{l}\text { Longitud } \\
\text { total de la } \\
\text { hoja }(\mathrm{cm})\end{array}$ & $\begin{array}{l}\text { Longitud } \\
\text { del peciolo } \\
\text { (cm) }\end{array}$ & $\begin{array}{l}\mathrm{N}^{\circ} \text { pares } \\
\text { de pinnas } \\
\text { primarias }\end{array}$ & $\begin{array}{c}\text { Longitud } \\
\text { de la } 1^{\circ} \\
\text { pinna }(\mathrm{cm})\end{array}$ & $\begin{array}{c}\text { Ancho de } \\
\text { la } 1^{\circ} \text { pinna } \\
(\mathrm{cm})\end{array}$ & $\begin{array}{l}\mathrm{N}^{\circ} \text { pares } \\
\text { de foliolos } \\
1^{\circ} \text { pinna }\end{array}$ & $\begin{array}{l}\text { Dist entre } \\
\text { foliolos } \\
(\mathrm{mm}) 1^{\circ} \\
\text { pinna }\end{array}$ & $\begin{array}{l}\text { Largo de } \\
\text { foliolo } \\
(\mathrm{mm}) 1^{\circ} \\
\text { pinna }\end{array}$ & $\begin{array}{l}\text { Ancho de } \\
\text { foliolo } 1^{\circ} \text { pinna } \\
(\mathrm{mm})\end{array}$ \\
\hline 3073 & 6,80 & 1,25 & 2,75 & 3,18 & 1,13 & 9,67 & 3,44 & 6,42 & 1,59 \\
\hline 3074 & 7,30 & 1,05 & 2,75 & 3,82 & 1,44 & 10,29 & 4,05 & 7,13 & 1,66 \\
\hline 3075 & 4,60 & 0,80 & 2,00 & 3,03 & 1,19 & 9,13 & 3,67 & 6,56 & 2,10 \\
\hline 3076 & 6,25 & 1,00 & 2,25 & 3,71 & 1,38 & 11,42 & 3,25 & 7,48 & 2,40 \\
\hline 3077 & 4,38 & 0,73 & 2,00 & 2,96 & 1,40 & 10,00 & 3,15 & 6,96 & 1,93 \\
\hline 3078 & 5,53 & 1,13 & 2,00 & 3,19 & 1,31 & 8,63 & 3,88 & 7,44 & 2,46 \\
\hline 3079 & 3,90 & 0,71 & 2,00 & 2,29 & 1,03 & 9,50 & 2,50 & 5,08 & 1,36 \\
\hline 3080 & 4,80 & 0,88 & 2,00 & 2,95 & 1,60 & 8,75 & 3,63 & 6,41 & 1,61 \\
\hline 3081 & 5,53 & 1,18 & 2,00 & 3,20 & 1,03 & 10,13 & 3,06 & 5,19 & 1,68 \\
\hline 3082 & 4,63 & 0,73 & 2,50 & 2,81 & 1,15 & 9,92 & 3,06 & 5,66 & 1,61 \\
\hline 3083 & 5,13 & 1,10 & 2,75 & 2,87 & 1,05 & 10,08 & 2,79 & 5,35 & 1,55 \\
\hline 3084 & 4,10 & 0,63 & 3,00 & 2,23 & 0,85 & 8,83 & 2,63 & 4,51 & 1,16 \\
\hline 3085 & 4,60 & 0,80 & 2,50 & 2,75 & 1,08 & 10,92 & 2,67 & 5,30 & 1,38 \\
\hline 3086 & 5,75 & 0,88 & 2,75 & 2,93 & 1,05 & 10,79 & 3,00 & 5,84 & 1,43 \\
\hline 3087 & 4,35 & 0,95 & 2,25 & 2,47 & 0,87 & 11,21 & 2,33 & 4,72 & 1,16 \\
\hline 3089 & 6,50 & 1,48 & 2,00 & 4,15 & 1,54 & 10,63 & 4,10 & 7,88 & 3,00 \\
\hline 3092 & 5,88 & 1,13 & 2,25 & 3,29 & 1,29 & 11,75 & 2,98 & 6,63 & 1,81 \\
\hline 3094 & 7,68 & 1,40 & 2,25 & 4,37 & 1,40 & 12,75 & 3,70 & 7,06 & 2,23 \\
\hline 3095 & 6,33 & 1,03 & 2,00 & 3,78 & 1,44 & 9,88 & 4,08 & 7,31 & 1,93 \\
\hline 3096 & 6,00 & 0,48 & 3,00 & 3,25 & 1,07 & 10,65 & 3,50 & 5,41 & 1,55 \\
\hline 3097 & 4,43 & 0,83 & 2,00 & 2,36 & 1,00 & 9,88 & 2,75 & 5,44 & 1,43 \\
\hline 3098 & 5,35 & 1,05 & 2,25 & 3,00 & 1,25 & 10,46 & 3,50 & 6,27 & 1,50 \\
\hline 3101 & 6,43 & 0,85 & 2,50 & 3,89 & 1,41 & 11,46 & 3,98 & 7,17 & 2,27 \\
\hline 3110 & 7,13 & 1,90 & 2,00 & 4,13 & 1,61 & 10,13 & 4,46 & 8,46 & 2,66 \\
\hline $3287-58$ pa & 9,36 & 2,08 & 3,00 & 3,36 & 1,36 & 10,60 & 2,60 & 7,60 & 2,00 \\
\hline 3288-59pax & 6,86 & 0,90 & 3,00 & 3,02 & 1,08 & 10,00 & 2,00 & 5,40 & 1,80 \\
\hline 3289-60pa & 9,38 & 2,00 & 2,40 & 4,80 & 1,42 & 10,40 & 3,40 & 9,20 & 3,20 \\
\hline 3290-61pa & 8,96 & 1,52 & 2,80 & 3,46 & 1,48 & 8,60 & 3,60 & 7,80 & 2,40 \\
\hline $3291-62$ & 6,94 & 1,46 & 2,60 & 2,60 & 1,02 & 7,00 & 3,40 & 4,80 & 1,40 \\
\hline $3292-63$ & 8,02 & 1,36 & 2,60 & 4,02 & 1,76 & 9,80 & 2,40 & 8,00 & 3,00 \\
\hline 3293-64pa & 10,34 & 2,72 & 2,60 & 4,18 & 1,42 & 11,40 & 3,20 & 7,80 & 2,80 \\
\hline 3294-65pa & 10,44 & 2,22 & 2,40 & 5,44 & 1,96 & 11,80 & 3,60 & 9,80 & 3,00 \\
\hline 3295-66pa & 8,22 & 1,90 & 2,60 & 4,50 & 1,74 & 12,20 & 2,80 & 9,80 & 2,60 \\
\hline $3296-67$ & 8,26 & 2,18 & 2,00 & 4,66 & 1,68 & 11,40 & 4,00 & 8,80 & 2,20 \\
\hline 3297-68pa & 8,82 & 2,76 & 2,80 & 3,48 & 1,84 & 10,00 & 2,40 & 9,20 & 2,00 \\
\hline 3298-69pa & 9,42 & 2,28 & 2,20 & 4,24 & 1,98 & 10,80 & 2,60 & 10,00 & 3,20 \\
\hline 3299-70pa & 6,94 & 1,18 & 2,20 & 3,46 & 1,52 & 10,20 & 3,00 & 7,80 & 2,20 \\
\hline 3300-71 & 8,04 & 2,28 & 2,40 & 5,22 & 2,12 & 12,40 & 3,60 & 9,80 & 2,80 \\
\hline 3301-72pa & 10,02 & 2,16 & 2,80 & 5,20 & 1,88 & 11,80 & 3,80 & 9,40 & 2,60 \\
\hline 3302-73pa & 8,00 & 1,38 & 2,20 & 4,06 & 1,42 & 11,60 & 3,00 & 7,20 & 2,00 \\
\hline $3303-74 \mathrm{pa}$ & 8,20 & 2,24 & 2,20 & 3,72 & 1,50 & 13,00 & 2,20 & 7,20 & 2,00 \\
\hline $3304-75 \mathrm{pa}$ & 9,66 & 1,94 & 3,00 & 4,22 & 1,82 & 13,80 & 2,40 & 9,60 & 2,60 \\
\hline 3305-76ра & 7,68 & 1,43 & 2,50 & 3,05 & 1,33 & 8,75 & 3,00 & 7,50 & 2,25 \\
\hline 3306-77pa & 9,20 & 2,16 & 2,80 & 3,54 & 1,48 & 9,80 & 2,60 & 7,20 & 2,20 \\
\hline $3307-78$ pa & 9,06 & 1,68 & 2,60 & 4,88 & 1,86 & 13,20 & 3,00 & 9,20 & 3,00 \\
\hline 3308-79?? & 11,80 & 1,96 & 2,40 & 6,16 & 2,32 & 12,20 & 4,40 & 11,40 & 3,40 \\
\hline 3309-80?? & 9,98 & 1,74 & 2,80 & 3,88 & 1,70 & 11,80 & 2,80 & 8,80 & 2,60 \\
\hline 3310-81?? & 11,04 & 3,12 & 2,40 & 5,70 & 2,24 & 11,80 & 4,00 & 10,80 & 3,60 \\
\hline 3311-82?? & 9,28 & 1,62 & 2,40 & 4,28 & 1,58 & 12,20 & 2,40 & 8,20 & 3,00 \\
\hline 3312-83?? & 11,48 & 2,18 & 2,60 & 4,46 & 1,58 & 10,60 & 3,00 & 7,80 & 2,60 \\
\hline 3313-84?? & 10,30 & 2,48 & 2,00 & 4,62 & 1,80 & 10,80 & 3,60 & 8,80 & 2,80 \\
\hline 3314-85?? & 13,62 & 3,20 & 2,00 & 6,78 & 2,02 & 13,00 & 4,00 & 10,00 & 3,80 \\
\hline $3315-86$ & 13,86 & 2,06 & 2,80 & 6,54 & 2,02 & 12,40 & 4,40 & 10,60 & 2,60 \\
\hline 3316-87?? & 9,86 & 1,88 & 3,00 & 4,36 & 1,62 & 10,20 & 3,00 & 7,40 & 2,20 \\
\hline 3317-88?? & 11,20 & 2,56 & 2,80 & 4,64 & 1,62 & 11,20 & 3,00 & 8,20 & 2,80 \\
\hline $3277 \mathrm{ch}$ & 13,81 & 2,87 & 1,13 & 10,94 & 2,94 & 21,86 & 4,60 & 14,70 & 1,54 \\
\hline $3276 \mathrm{ch}$ & 18,70 & 5,45 & 1,00 & 13,25 & 5,34 & 18,00 & 5,44 & 26,70 & 2,20 \\
\hline
\end{tabular}

(Continúa....) 
Apéndice 1. Continuación.

\begin{tabular}{|c|c|c|c|c|c|c|c|c|c|}
\hline $\mathrm{N}^{\circ}$ & $\begin{array}{l}\text { Longitud } \\
\text { total de la } \\
\text { hoja }(\mathrm{cm})\end{array}$ & $\begin{array}{l}\text { Longitud } \\
\text { del peciolo } \\
\text { (cm) }\end{array}$ & $\begin{array}{l}\mathrm{N}^{\circ} \text { pares } \\
\text { de pinnas } \\
\text { primarias }\end{array}$ & $\begin{array}{l}\text { Longitud } \\
\text { de la } 1^{\circ} \\
\text { pinna }(\mathrm{cm})\end{array}$ & $\begin{array}{l}\text { Ancho de } \\
\text { la } 1^{\circ} \text { pinna } \\
(\mathrm{cm})\end{array}$ & $\begin{array}{l}\mathrm{N}^{\circ} \text { pares } \\
\text { de foliolos } \\
1^{\circ} \text { pinna }\end{array}$ & $\begin{array}{l}\text { Dist entre } \\
\text { foliolos } \\
(\mathrm{mm}) 1^{\circ} \\
\text { pinna }\end{array}$ & $\begin{array}{l}\text { Largo de } \\
\text { foliolo } \\
(\mathrm{mm}) 1^{\circ} \\
\text { pinna }\end{array}$ & $\begin{array}{l}\text { Ancho de } \\
\text { foliolo } 1^{\circ} \text { pinna } \\
(\mathrm{mm})\end{array}$ \\
\hline $3275 \mathrm{ch}$ & 15,75 & 2,92 & 1,00 & 12,83 & 4,20 & 17,75 & 6,86 & 21,00 & 2,00 \\
\hline $3274 \mathrm{ch}$ & 17,01 & 5,35 & 1,00 & 11,66 & 4,02 & 21,00 & 6,18 & 20,10 & 1,95 \\
\hline $3278 \mathrm{ch}$ & 16,04 & 3,36 & 1,00 & 12,68 & 2,96 & 22,80 & 3,40 & 14,80 & 1,73 \\
\hline 3283pa & 6,33 & 1,86 & 2,00 & 3,52 & 1,50 & 10,12 & 3,37 & 7,50 & 1,93 \\
\hline 3284 pa & 4,74 & 0,98 & 2,00 & 2,28 & 1,44 & 9,33 & 2,66 & 7,20 & 2,00 \\
\hline 3286li & 2,87 & 1,00 & 2,80 & 2,50 & 1,04 & 11,13 & 1,07 & 5,22 & 1,11 \\
\hline 3282li & 4,40 & 0,87 & 2,57 & 2,17 & 0,86 & 10,33 & 1,00 & 4,30 & 1,00 \\
\hline 3285li & 2,57 & 0,65 & 2,14 & 1,87 & 1,00 & 8,55 & 1,93 & 5,00 & 1,86 \\
\hline 3281li & 5,10 & 0,78 & 3,10 & 2,72 & 1,28 & 12,71 & 1,72 & 6,40 & 1,46 \\
\hline 3280li & 4,30 & 0,77 & 3,00 & 2,24 & 1,15 & 10,80 & 1,72 & 5,77 & 1,70 \\
\hline
\end{tabular}




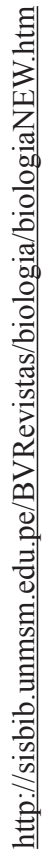

